\title{
LES INSTRUMENTS NON CONVENTIONNELS DE LA COMMISSION DU DROIT INTERNATIONAL DES NATIONS UNIES: SUCCES OU ECHEC DE LA CODIFICATION?
}

\author{
THE NON-CONVENTIONAL INSTRUMENTS OF THE UNITED \\ NATIONS' INTERNATIONAL LAW COMMISSION: SUCCESS OR \\ FAILURE OF CODIFICATION?
}

MAURIZIO ARCARI"

\begin{abstract}
RESUME
Une des tendances les plus remarquables des récents travaux de codification de la Commission du droit international des Nations Unies est l'adoption d'instruments non contraignants, tels que les projet de directives, de principes, de lignes-guide. Sur cette base, la doctrine a affirmé que l'outil classique de la codification, le traité, a été définitivement supplanté dans la pratique de la Commission. L'étude suggère que les instruments non contraignants ne sauraient toutefois devenir les outils exclusif dans l'œuvre de codification de la CDI. Ces instruments peuvent jouer une fonction positive quand il s'agit d'éclairer des aspects techniques du droit international ou d'intégrer certains aspects d'une discipline déjà consolidée. Par ailleurs, quand il s'agit de développer progressivement les règles substantielles du droit international dans un domaine donné, on ne pourrait pas facilement renoncer à l'élément de certitude inhérent aux traités de codification, et à l'expression du consentement des Etats qui en est à la base.
\end{abstract}

MOTS-CLÉS: Droit International. Commission du Droit International. Codification.

\begin{abstract}
One remarkable trend in the recent work of codification of the UN International Law Commission is the adoption of nonbinding instruments, such as draft directives, principles or guidelines. On this score, scholars suggest that the treaty, as a tool traditionally used for the codification of international law, has been definitively supplanted in the practice of the Commission. This article suggests that non-binding instruments cannot become the exclusive means available to the ILC for performing its tasks. Non-binding instruments can play a prominent role when the aim of the Commission is that of clarifying technical aspects of international law or that of supplementing already consolidated rules of international law. However, when the problem at stake is the progressive development of substantive rules of international law in a certain field, the legal certainty offered by codification treaties, as well as by the expression of the consent of States to be bound, can hardly be side-stepped.
\end{abstract}

KEYWORDS: International Law. International Law Commission. Codification.

\section{INTRODUCTION}

Si l'on donne un coup d'œil cursif à la littérature juridique contemporaine concernant les travaux de codification de la Commission du droit international des Nations Unies ('CDI' ou 'la Commission') il est aisé d'y trouver deux affirmations récurrentes. La première consiste à affirmer que le produit classique, " par excellence », des travaux de codification de la Commission, qui donnerait

\footnotetext{
* Professeur de droit international, Université Milano-Bicocca.

E-mail: maurizio.arcari@unimib.it
} 
aussi la mesure du succès de ses travaux, est le format conventionnel, le traité. La deuxième affirmation consiste à dire que le traité, dans le même temps, n'est pas la seule issue possible de ces travaux, d'autres instruments de codification à la valeur juridique moins forte étant aussi disponibles. A cet égard, force est de constater que de tels instruments de " codification molle ${ }^{1}$ sont en train de prendre le pas dans l'expérience récente de la CDI.

Pour donner une idée de l'évolution du phénomène, on peut utilement se référer à un article de David Caron concernant les rapports entre forme et autorité dans le Projet d'articles de la CDI sur la responsabilité internationale de l'Etat. L'auteur qualifiait les projets de traités élaborés par la CDI ( Draft conventions») comme le style de travail dominant à la CDI (" the dominant working style of the ILC »), tout en admettant que celle-ci pourrait également choisir et utiliser d'autres formats pour les produits finaux de ses travaux ( «It [=la CDI] could use other forms of documents and could look to other final products " $)^{2}$. D'autre part, dans une note bas de page, l'auteur ajoutait que, nonobstant le recours croissants à ces instruments alternatifs de codification, il n'était pas à même de prévoir une déviation réelle de la tendance dominante de la CDI concernant l'utilisation de la forme conventionnelle ${ }^{3}$.

Toutefois, si on considère la pratique de la codification aux Nations Unies dans le nouveau millénaire, on peut légitimement affirmer que la tendance est à l'heure actuelle renversée et que les « instruments non conventionnels » représentent maintenant le choix préférentiel de la CDI quant à la forme finale à donner aux travaux de codification. Les données répertoriées dans l'étude de Laurence Helfer et Timothy Meyer sont à cet égard très révélatrices ${ }^{4}$. Parmi les 30 projets d'articles complétés entre 1949 et 1999, 20 ont été recommandés par la CDI à l'Assemblée générale (AG) pour la consécration dans un traité. Par contre, sur les 12 projets d'articles menés à bien entre 2000 et 2014, deux fois seulement la CDI a demandé l'élaboration d'une convention (s'agissant des projets sur la Prévention des dommages transfrontières résultant d'activités dangereuses ${ }^{5}$ et sur la Protection diplomatique ${ }^{6}$ ), tandis que dans les autres cas

1 Pour l'utilisation de cette expression voir PELLET, Alain. Conclusions. In : Société Française pour le Droit International, Colloque d'Aix en Provence. La codification du droit international. Paris : Pédone, 1999, p. 337.

2 Cf. CARON, David. The ILC Articles on State Responsibility: The Paradoxical Relationship between Form and Authority. In: American Journal of International Law, 96 (2002), p. 862.

3 Ibidem, note bas de page 27 : "Although there are some exceptions, I do not see a trend away from the dominant form. However, it is difficult to draw a strong conclusion ".

4 HELFER, Laurence R., MEYER, Timothy. The Evolution of Codification. A Principal-Agent Theory of the International Law Commission's Influence. In: BRADLEY, Curtis A. (ed.). Custom's Future. International Law in a Changing World. Cambridge: Cambridge University Press, 2016, pp. 315-317.

5 Annuaire de la Commission du droit international, vol. II/2, 2001, p. 156, par. 94.

6 Rapport de la Commission du droit international, Cinquante-huitième session 1 mai-9 juin et 
la Commission a préféré recommander à l'organe politique des Nations Unies de " prendre acte du projet d'articles ... dans une résolution et d'annexer ces articles à la résolution » (projet d'articles sur le Droit des aquifères transfrontières ${ }^{7}$ ), de " prendre note » de l'instrument en question et " d'en assurer la plus large diffusion " (Guide de la pratique sur les réserves aux traités ${ }^{8}$ ), ou s'est tout simplement limité à " recommander » le texte à l'attention de l'AG (Principes directeurs applicables aux déclarations unilatérales des Etats susceptibles de créer des obligations juridiques ${ }^{9}$ ). Cette tendance semble confirmée par la lecture du plus récent des rapports de la CDI. Sur les huit sujets à l'attention de la Commission pendant la session de 2019, cinq sont conçus sous forme d'instruments non contraignants (qu'il agit d'un projet de «clause-type » ou de «conclusions » ou de «principes », ou tout simplement d'un rapport d'un Groupe d'étude), tandis que trois seulement se présentent sous le format plus traditionnel $\mathrm{du}$ " projet d'articles ", dont la transformation en convention reste par ailleurs aléatoire ${ }^{10}$. D'emblée, cette inclination de la CDI à utiliser de nouvelles solutions pour ses travaux a amené les commentateurs à constater que « codification appears to have simply shifted away from the conventional form " ${ }^{11}$, et à conclure que " what

3 juillet-11 août 2006, UN doc. A/61/10 (2006), p. 15, par. 46.

7 Rapport de la Commission du droit international, Soixantième session 5 mai-6 juin et 7 juillet-8 août 2008, UN doc. A/63/10 (2008), p. 19, par. 49.

8 Rapport de la Commission du droit international, Soixante-troisième session 26 avril-3 juin et 4 juillet-12 août 2011, UN doc. A/66/10 (2011), p. 17, par. 72.

9 UN doc. A/61/10 (2006) cit., p. 384, par. 170.

10 Voir Rapport de la Commission du droit international, Soixante et onzième session 29 avril-7 juin et 8 juillet-9 aô̂t 2019, UN doc. A/74/10 (2019), p. 4, par. 11, pour la liste des sujets inscrits dans le programme de travail de la CDI. En particulier, les sujets Normes impératives $d u$ droit international général (jus cogens) et Principes généraux du droit se présentent sous la forme du projet de conclusions (le projet sur le premier thème ayant été adopté en 2019 en première lecture, le deuxième étant dans la phase initiale d'élaboration : ibidem, respectivement par. 53-54 et par. 234) ; le sujet Protection de l'environnement en rapport avec les conflits armés a été adopté en première lecture par la Commission en 2019 sous la forme de projet de principes (ibidem, par. 66-68) ; le sujet L'élévation du niveau de la mer au regard du droit international est à l'heure actuelle à l'attention d'un groupe d'étude de la CDI (par. 265-273) ; concernant le sujet Application provisoire des traités, sur lequel un projet de directives avait été adopté en première lecture en 2018, la CDI a inclus dans le rapport de 2019 un texte révisé de projets de clause-type, en vue de demander aux Etats leurs commentaires (ibidem, par. 284). Par contre, les sujets Crimes contre l'humanité, Immunité de juridiction pénale étrangère des représentants de l'Etat et Succession d'Etats en matière de responsabilité internationale de l'Etat se présentent sous la forme plus classique du projet d'articles. Toutefois, à la session de 2019 la CDI a recommandé à l'AG le projet d'articles sur la prévention et la prévention des crimes contre l'humanité, en préconisant l'adoption d'une convention sur cette base (ibidem, par. 42) ; pendant la même session, la décision de faire aboutir ou non le projet d'articles sur l'immunité de juridiction pénale étrangère des représentants de l'Etat sous forme d'un traité a été considérée comme prématurée (cf. ibidem, par. 184 et 201) ; enfin, une attitude possibiliste a été retenue sur la question de la forme finale du projet d'articles sur la succession d'Etats en matière de responsabilité internationale (ibidem, par. 105).

11 VIllalPANDO, Santiago. Codification Light: A New Trend in the Codification of International Law at the United Nations. In: Anuário Brasileiro de Direito Internacional, 8 
before was considered a failure (the refusal of the GA to pursue a convention) is now envisioned as a goal $" .{ }^{12}$

Dans la suite de cette étude, on tentera d'explorer certaines des raisons qui justifient cette "nouvelle vague » des travaux de la CDI. Tout en évitant de se plonger dans l'analyse détaillée des différents instruments non-conventionnels adoptés par la CDI, on s'occupera tout d'abord d'exposer les traits généraux de la catégorie des « instruments non-conventionnels » et de mettre en exergue que cette catégorie est loin d'être homogène, puisqu'elle réunit des instruments différenciés quant au contenu et aux finalités (2). Ensuite, on tentera d'esquisser un bilan de l'œuvre accomplie par la CDI avec les instruments non conventionnels, en s'arrêtant sur une évaluation des éléments qui contribuent à déterminer le succès de ces instruments, ainsi que de leurs aspects problématiques (3). Finalement, on formulera quelques remarques de caractère conclusif sur l'impact des instruments non-conventionnels sur le processus de codification du droit international et sur le rôle de la CDI dans ce domaine (4).

\section{LES INSTRUMENTS NON-CONVENTIONNELS DE CODIFI- CATION, UNE CATEGORIE HETEROGENE ET ADAPTABLE}

Un premier constat, somme toute banal, est que la catégorie des " instruments non-conventionnels » proposés par la CDI n'est ni nouvelle, ni révolutionnaire. Le Statut de la CDI envisage, à l'art. 23, une pluralité de solutions que la Commission peut recommander à l'AG comme issue de ses travaux. A côté de la conclusion d'une convention ou de la convocation d'une conférence diplomatique à cet effet, la possibilité de n'entreprendre aucune action, celle de prendre acte seulement du rapport de la Commission, ou celle de l'adopter dans une résolution, sont mentionnées ${ }^{13}$. La pratique des premières cinquante années de vie de la CDI offre d'autre part un certain nombre d'exemples d'instruments s'inscrivant dans la logique de la codification " non-conventionnelle ", comme par exemple le Projet de déclaration sur les droits et les devoirs des Etats de $1949^{14}$, les Principes de droit international consacrés par le Statut du Tribunal de

(2013), p. 118.

12 KATZ COGAN, Jacob. The Changing Form of the International Law Commission's Work. In: VIRZO, Roberto, INGRAVALLO, Ivan (eds.). Evolutions in the Law of International Organizations. Boston/Leiden: Brill/Nijhoff, 2015, p. 283.

13 L'art. 23, par. 1, du Statut de la CDI énonce : «La Commission peut recommander à l'Assemblée générale : a) De n'entreprendre aucune action, le rapport ayant été publié ; $b$ ) De prendre acte du rapport, ou de l'adopter dans une résolution ; c) De recommander le projet aux Membres en vue de la conclusion d'une convention ; $d$ ) de convoquer une conférence pour conclure une convention".

14 Voir Rapport de la Commission du droit international, Première session 12 avril-9 juin 1949, UN doc. A/925 (1949), notamment par. 53, dans lequel la Commission décida de soumettre à l'AG le Projet de Déclaration et de laisser à celle-ci la décision sur l'action à prendre. 
Nuremberg et dans le jugement de ce Tribunal de $1950^{15}$ ou le Modèle de règles sur la procédure arbitrale de $1958^{16}$. Cependant, comme on l'a déjà noté dans les considérations introductives, c'est après le tournant du XXIème siècle que la nouvelle pratique de la codification non-conventionnelle a rebondi, en ajoutant aux possibilités déjà connues un nombre de solutions inédites, dont l'originalité est directement suggérée par l'intitulé de l'instrument en question. En sont des bons exemples des textes achevés comme les Conclusions du Groupe d'étude de la CDI sur la fragmentation du droit international $(2006)^{17}$, les Principes directeurs applicables aux déclarations unilatérales des Etats $(2006)^{18}$, le Projet de principes sur la répartition des pertes en cas de dommage transfrontière découlant d'activités dangereuses $(2006)^{19}$, le Guide de la pratique des réserves aux traités $(2011)^{20}$, le Rapport final du Groupe de travail sur l'obligation d'extrader ou de poursuivre (aut dedere aut indicare) (2014) $)^{21}$, les projets de conclusions sur les Accords et pratique ultérieurs dans le contexte de l'interprétation des traités (2018) et sur la Détermination du droit international coutumier $(2018)^{22}$, auxquels vont

15 Voir Rapport de la Commission du droit international, Deuxième session 5 juin-29 juillet 1950, UN doc. A/1316 (1950), par. 97 s. A cette occasion, la CDI s'est limitée à adopter les Principes, sans adresser aucune recommandation à l'AG.

16 Voir Annuaire de la Commission du droit international, 1958, vol. II, p. 83 s., notamment par. 13, où la CDI avalise le choix de réserver aux articles sur la procédure arbitrale la valeur de modèle de règles.

17 Pendant sa cinquante-huitième session de 2006 la CDI a pris note des 42 conclusions du Groupe de travail sur la fragmentation et les recommandées à l'AG (UN doc. A/66/10 (2006), par. 239). Le texte du rapport final du Groupe de travail est reproduit dans le document Fragmentation $d u$ droit international : difficultés découlant de la diversification et de l'expansion du droit international. Rapport du Groupe d'étude de la Commission du droit international. Etabli sous sa forme définitive par Martti Koskenniemi, UN doc. A/CN.4/L.682 du 13 avril 2006.

18 Voir références supra, note 9.

19 A sa cinquante-huitième session de 2006 la CDI a recommandé à l'AG d'entériner les projets de principes sous forme d'une résolution et de prier instamment les Etats d'agir aux niveaux national et international afin de mettre en œuvre lesdits principes : UN doc. A/61/10 (2006) cit., par. 63 et par. 66 s., pour le texte des principes.

20 Voir références supra, note 8 et, pour le texte du Guide, UN doc. A/66/10/Add.1 (2011), p. 2 s. Pour un commentaire concernant le rôle de cet instrument de codification, voir CASSELLA, Sarah. Le Guide de la pratique sur les réserves aux traités : une nouvelle forme de codification? In : Annuaire Français de Droit International, 58 (2012), p. 29 s.

21 Pendant sa session de 2014, la CDI a adopté le rapport final sur le sujet et a décidé d'en clore l'examen : voir Rapport de la Commission du droit international, Soixante-sixième session 5 mai-6 juin et 7 juillet-8 aô̂t 2014, UN doc. A/69/10 (2014), par. 64 pour les décisions de la Commission et par 65 s. pour le texte du rapport final.

22 Les deux projets de conclusions ayant été adoptés en deuxième lecture pendant la session de 2018, la CDI a recommandé à l'Assemblée générale a) de prendre note des projets d'articles dans une résolution, de les annexer à celle-ci et d'en assurer la plus ample diffusion possible ; b) de recommander les projets de conclusions et les commentaires y relatifs à l'attention des Etats et des toutes les personnes qui peuvent être appelées respectivement à interpréter les traités ou à déterminer l'existence de règles du droit international coutumier: voir Rapport de la Commission du droit international, Soixante-dixième session 30 avril-1 $1^{\text {er }}$ juin et 2 juillet-10 aôut 2018, UN doc. A/73/10 (2018), respectivement par. 46-49 et 58-63. 
s'ajouter, en perspective, d'autres sujets en cours d'élaboration comme le projet de conclusions sur la Protection de l'atmosphère ${ }^{23}$ ou le projet de conclusions sur les Normes impératives du droit international général (ius cogens) ${ }^{24}$.

Par ailleurs, non seulement la catégorie des instruments non-conventionnels proposés par la CDI est loin d'être nouvelle, mais elle est au surplus hétérogène, et ceci bien au-delà des intitulés choisis pour les textes qui la composent. Il faut en effet observer que - à côté des différents " guides ", " principes directeurs ", « conclusions ", " rapports » susmentionnés, qui déjà à partir de la dénomination indiquent leur vocation à garder le statut d'actes non contraignants - on peut aussi inclure dans la catégorie des instruments non-conventionnels une autre panoplie de textes plus classiquement désignés par la CDI comme " projet d'articles », mais par rapport auxquels la transformation en traité peut être d'emblée exclue ou, tout en n'étant pas exclue a priori, est seulement envisagée comme l'une des issues futures et potentielles. On pourrait indiquer en exemples de la première possibilité le projet d'articles sur le Droit des aquifères transfrontières, par rapport auquel la CDI elle-même a été explicite dans le sens de l'exclusion du format conventionnel, en demandant à l'AG tout simplement de prendre note du Projet dans une résolution et d'annexer les articles à la résolution ${ }^{25}$. Mais bien plus connues sont les solutions " possibilistes " élaborées en référence aux deux projets d'articles sur la Responsabilité de l'Etat pour fait internationalement illicite (2001) et sur la Responsabilité des Organisations internationales (2011), à propos desquels la CDI a recommandé à l'AG de " prendre acte » des projets et d'envisager à un stade ultérieur la possibilité de la convocation d'une conférence diplomatique en vue de l'adoption de conventions dans la matière ${ }^{26}$. Cette solution a été aussi réitérée pour le projet d'articles sur les Effets des conflits armés sur les traités $(2011)^{27}$ et le projet d'articles sur l'Expulsion des étrangers $(2014)^{28}$. Or, il est vrai que, à strictement parler, il ne s'agit pas d'instruments pour lesquels la CDI a directement et ouvertement proposé le format non-conventionnel : celui-ci reste seulement une des possibilités envisagées, car la recommandation adressée par la CDI à l'AG ne fait pas obstacle à la possibilité que les projets

23 Le texte des projets de directive sur la Protection de l'atmosphère a été adopté en première lecture par la CDI pendant sa session de 2018 : cf. ibidem, par. 73 s.

24 Le projet de conclusions sur les Normes impératives du droit international général a été adopté en première lecture par la CDI à la session de 2019 ; rappelons que, à la session de 2019, la CDI a aussi adopté en première lecture le projet de principes sur la Protection de l'environnement en rapport avec les conflits armés (voir références supra, note 10).

25 Voir références supra, note 7.

26 Voir respectivement les conclusions de la CDI dans Annuaire de la Commission du droit international, 2001, vol II/2, p. 25, par. $72-73$ (concernant le projet d'articles sur la responsabilité des Etats) et UN doc. A/66/10 (2011) cit., par. 85 (concernant le projet sur la responsabilité des Organisations internationales).

27 Voir UN doc. A/66/10 (2011) cit., par. 97.

28 Voir UN doc. A/69/10 (2014) cit., par. 42. 
d'articles puissent se transformer, dans un futur plus ou moins proche, dans un traité. Néanmoins, le fait que l'AG (par ailleurs suivant l'indication donnée par la CDI elle-même) ait pris l'habitude d'entériner ces projets dans une résolution à laquelle le texte des articles est annexé, contribue à donner à ces documents une certaine allure finalisée. Comme différents auteurs l'ont noté, de ce point de vue le fait que le texte des résolutions pertinentes de l'AG ne se réfère pas $\mathrm{au}$ " projet d'articles », mais tout simplement aux " articles » de la CDI semble représenter plus qu'un simple élément formel, et corrobore l'impression que le texte de la CDI se trouve avalisé et figé par l'AG dans son statut d'instrument non-conventionnel et non-contraignant ${ }^{29}$.

Etant donnée l'hétérogénéité des textes susceptibles d'être réunis dans la catégorie des instruments non-conventionnels, on pourrait se poser la question si le recours par la CDI à cette typologie d'instruments est susceptible de répondre à une logique cohérente ou si, au contraire, il est le produit de choix occasionnels, faits au cas par cas. Comme justement noté dans la doctrine, il est en effet difficile d'envisager une approche unitaire à cet égard, car le recours aux instruments de la « codification molle » répond souvent aux exigences du moment, du sujet et du contenu qui sont propres à chaque entreprise de codification ${ }^{30}$. Il paraît toutefois possible d'atténuer le relativisme de cette conclusion, dans le sens que dans certains cas l'option de la CDI pour l'instrument non contraignant pourrait être a priori liée au contenu de la matière objet de codification et aux finalités que l'œuvre codificatrice se propose. Tel serait le cas des travaux de la CDI dont le propos est de compléter, clarifier ou intégrer certains aspects d'une codification déjà achevée, plutôt que de proposer des instruments nouveaux ou des règles inédites. Que l'on songe par exemple, parmi les projets " achevés », au guide

29 Voir par exemple les remarques de LUSA BORDIN, Fernando. Reflections of Customary International Law: The Authority of Codification Conventions and ILC Draft Articles in International Law. In: International and Comparative Law Quarterly, 63 (2014), p. 557: « The omission of the term 'draft' is of symbolic value and reinforces perceptions that the codification is a finalized project, thereby enhancing its claim to authority ». Dans le même sens aussi PRONTO, Arnold. Some Thoughts on the Making of International Law. In: European Journal of International Law, 19 (2008), p. 610 ; VILLALPANDO. Codification Light... cit., p. 118 ; KATZ COGAN. The Changing Form ... cit., pp. 287-289.

30 Voir en ce sens PELLET. Conclusions cit., p. 333 : «tout dépend du sujet, du moment et du contenu »; TOMUSCHAT, Christian. L'exemple de la Commission du droit international. In : Société Française de Droit International, Colloque d'Aix en Provence. La codification du droit international. Paris : Pédone, 1999, p. 190, selon lequel « En ce qui concerne le produit final d'un processus législatif entamé par la CDI (...) il est impossible d'y porter un jugement général. (...) Ce sont toujours les particularités de l'espèce qui devraient l'emporter ». Voir aussi l'observation de synthèse très efficace de WOOD, Michael. The General Assembly and the International Law Commission: What Happens to the Commission's Work and Why? In: BUFFARD, Isabelle, CRAWFORD, James, PELLET, Alain, WITTICH, Stephan (eds.). International Law between Universalism and Fragmentation. Festschrift in Honour of Gerhard Hafner. Leiden/Boston: Martinus Nijhoff Publishers, 2008, p. 383 : "The principal consideration is the subject-matter ». 
de la Pratique des réserves aux traités, aux conclusions concernant les Accords et pratique ultérieurs dans le contexte de l'interprétation des traités, ou encore, parmi les travaux en cours, au projet de clause-type concernant l'Application provisoire des traités. Dans ces cas, le propos de ne pas remettre en discussion l'acquis normatif représenté par la Convention de Vienne sur le droit des traités de 1969 est clair et bien établi dès le début de l'entreprise et il est tout à fait logique admettre que de la CDI, dans le développement de son propos, ne pourra que privilégier le format non conventionnel et non-contraignant ${ }^{31}$. Ce format serait en effet à même de garantir les exigences de flexibilité et d'adaptabilité demandées à un ouvrage de codification dont le but est d'intégrer ou éclairer une matière conventionnelle préexistante, sans pour autant en remettre en discussion le statut $^{32}$. Mais des conclusions similaires sembleraient s'imposer aussi par rapport à d'autres œuvres de codification qui, tout en ne s'appuyant pas sur des traités préexistants, se proposent d'éclairer les aspects techniques du fonctionnement du droit international et de ses règles, et par là de fournir des modèles de référence aux opérateurs de ce droit, comme dans le cas des conclusions sur la Fragmentation $d u$ droit international ${ }^{33}$, du projet de conclusion sur la Détermination $d u d r o i t$ international coutumier ${ }^{34}$ ou encore du projet de conclusion sur les Normes

31 Voir, par exemple, par rapport au sujet des réserves aux traités, l'approche retenue par la CDI pendant sa session de 1995 : «Les membres de la Commission ont tous souscrit à l'opinion du Rapporteur spécial selon laquelle il n'y avait pas lieu, en principe, de remettre en question les textes issus de la deuxième session de la Conférence des Nations Unies sur le droit des traités. Ils sont convenus que la Commission devait se borner à s'efforcer d'en combler les lacunes et d'en lever les ambiguïtés, mais en préservant l'adaptabilité et la souplesse des articles essentiels des Conventions de Vienne de 1969, 1978 et 1986 " (Annuaire de la Commission du droit international, 1995, vol. II/2, pp. 108-109, par. 439). Voir aussi les paragraphes 2 et 5 de l'introduction au Guide de la pratique adopté par la CDI en 2011, dans UN doc. A/66/10/ Add. 1 cit., pp. 38-40.

32 Voir PELLET, Alain. La Commission du droit international, pour quoi faire ? In : Paix, Développement, Démocratie. Boutros Boutros-Ghali Amicorum Discipulorumque Liber. Bruxelles : Bruylant, 1998, pp. 608-609, lequel à cet égard met en exergue le propos de « ne pas créer un imbroglio juridique en envisageant l'adoption d'un nouvel instrument conventionnel dont l'articulation avec les Conventions de Vienne (...) créerait une situation inutilement compliquée ».

33 Voir UN Doc. A/61/10 (2006) cit., par. 235, où la CDI présente les conclusions du Groupe de travail sur la fragmentation comme " une suite d'énoncés succincts, concrets et pragmatiques, conçus (...) comme un jeu de directives pratiques qui aideraient à examiner et à gérer la question de la fragmentation dans la pratique juridique ».

34 Voir le commentaire général de la CDI au projet de conclusions sur la détermination du droit coutumier : «Les présents projets de conclusion concernent le mode de détermination des règles du droit international coutumier. Ils visent à indiquer la voie à suivre pour déterminer l'existence de règles du droit international coutumier et leur contenu. (...) Partant du constat que le processus de détermination du droit international coutumier ne se prête pas toujours à des formulations précises, les projets de conclusion visent à indiquer la voie à suivre sans être excessivement prescriptifs » (UN doc. A/73/10 (2018) cit., pp. 129-130, par. 2 et 4 du commentaire). 
impératives du droit international général (jus cogens) 35 actuellement en instance. Nonobstant les variations possibles, il reste donc que le format non contraignant s'impose comme le plus adapté pour répondre aux besoins de l'œuvre codificatrice entamée par la Commission.

Par contre, dans d'autres cas, l'option de la CDI pour le format nonconventionnel ne semble pas représenter un choix cohérent avec le contenu et les finalités du texte envisagé. Cette option représente plutôt une réaction aux difficultés inhérentes à l'entreprise de codification et au caractère controversé des solutions retenues dans le projet élaboré ou en cours d'élaboration par la CDI. Ces difficultés peuvent découler du désaccord existant entre les membres de la Commission elle-même quant à la forme appropriée à réserver au produit final de la codification ${ }^{36}$, ou des perplexités exprimées par les Etats sur la question de la consécration sous forme conventionnelle d'un certain projet d'articles à l'étude de la Commission ${ }^{37}$. L'exemple déjà mentionné du compromis réalisé par rapport à l'issue des deux projets d'articles sur la responsabilité internationale est trop connu pour que l'on doive y revenir ${ }^{38}$. Quoi qu'il en soit, il est difficile de se cacher que dans ces hypothèses le recours à l'instrument non-conventionnel sera le produit, plus que d'une approche de fond mesurée sur le plan juridique et fonctionnelle à la matière objet de codification, de motivations de nature plus proprement politiques et occasionnelles, qui déconseillent de préjuger le sort $\mathrm{du}$ projet de codification en insistant sur les exigences de certitude juridique et de profiter, au contraire, de la flexibilité, voire de l'esprit de compromis, offerts par le format non-conventionnel. Ces dernières considérations nous portent à considérer les avantages et les limites respectives du format non-conventionnel, dans la perspective de vérifier si la tendance de la CDI consistant à privilégier ce

35 Voir le commentaire de la CDI à la conclusion 1 du projet adopté en première lecture à la session de 2019 : "Les présents projets de conclusion portent sur les normes impératives du droit international général (jus cogens), auxquelles font de plus en plus souvent référence les juridictions internationales et régionales, les juridictions nationales, les représentants des Etats et d'autres acteurs. Ils visent à fournir des orientations à tous ceux qui peuvent être appelés à déterminer l'existence des normes impératives du droit international général (jus cogens) et les conséquences juridiques de ces normes » (UN doc. A/74/10 (2019) cit., p. 156, par. 2 du commentaire).

36 Voir par exemple la division qui s'était déjà produite au sein de la CDI quant à la forme finale du projet d'articles sur la responsabilité internationale de l'Etat : Annuaire de la Commission $d u$ droit international, vol. II/2, pp. 24-25, par. 61-67.

37 Voir les perplexités exprimées par les Etats réunis à la Sixième Commission de l'AG quant au format du Projet d'articles sur l'expulsion des étrangers, telles que présentées dans le Résumé thématique des débats tenus par la Sixième Commission de l'Assemblée générale à sa soixanteneuvième session, établi par le Secrétariat, UN doc. A/CN.4/678 du 21 janvier 2015, par. 101. Pour un autre exemple, voir les commentaires des Etats sur le format du projet d'articles concernant les Effets des conflits armés sur les traités, résumés par le Rapporteur spécial Lucius Caflisch, Premier rapport sur les effets des conflits armés sur les traités, UN doc. A/CN.4/627/ Add.1 du 21 avril 2010, par. 153-154.

38 Voir supra, note 24 et texte relatif. 
format dans ses travaux récents peut être évaluée comme la manifestation d'un succès ou, au contraire, comme un échec de l'œuvre de codification.

\section{LES AVANTAGES ET LES LIMITES DES INSTRUMENTS NON CONVENTIONNELS DE CODIFICATION}

Les raisons militant pour le choix de l'instrument non-contraignant comme issue du processus de codification sont très bien connues. A cet égard, l'instrument non-contraignant est considéré de manière décisive comme permettant, grâce à sa flexibilité, de pallier certains des aspects les plus "épineux » qui sont associés à la conclusion d'un traité de codification. L'un des risques fréquemment évoqués à ce sujet est que le traité puisse figer indûment la règle coutumière qu'il entend codifier, en empêchant de cette manière les adaptations normatives qui se rendraient nécessaires en conséquence des évolutions de la vie des relations internationales. En outre, est allégué le danger de la soi-disant « décodification », résultant de la circonstance que, une fois adopté le texte du traité de codification à la suite d'une conférence diplomatique, celui-ci puisse recevoir un nombre de ratifications insuffisantes à son entrée en vigueur, avec la conséquence de faire plonger le droit qu'il se proposait de clarifier dans un état d'incertitude pire que celui existant avant le début du processus de codification ${ }^{39}$.

Enfin, un argument est apparu spécialement dans les phases finales de l'élaboration du projet d'articles sur la responsabilité internationale de l'Etat et qui présente une certaine force, dans la mesure où il fait appel aux exigences d'intégrité et d'efficacité du processus de codification. Cet argument s'appuie sur la possibilité que, spécialement dans le cas de projets d'articles portant sur des sujets complexes et controversés (tels que celui de la responsabilité internationale de l'Etat), qui ont été élaborés et finalement adoptés après des longues et laborieuses réflexions de la part de la Commission, l'ouverture ou la réouverture des négociations dans le contexte d'une conférence diplomatique risque de compromettre les équilibres atteints par l'organe technique et de remettre finalement en cause l'autorité globale de l'exercice de codification, ainsi que son utilité finale. Face à ce risque, on suggère alors de mettre le projet d'articles à l'abri des compromis politiques associés aux négociations interétatiques, en gardant l'intégrité des solutions normatives consacrées dans les textes de la CDI, dont l'équilibre serait garanti par l'indiscutable compétence technique de l'organe ${ }^{40}$.

39 Pour un aperçu de ces difficultés et des différentes positions doctrinales à cet égard, voir SINCLAIR, Ian. The International Law Commission. Cambridge : Grotius Publications Limited, 1987, pp. 138-145, sous la rubrique "The desirability of codification and its relationship to customary international law ». Voir aussi, plus récemment, l'excellent résumé de LUSA BORDIN. Reflections... op. cit., pp. 538-542.

40 Pour un exposé de ces arguments, avancés au sujet du projet d'articles sur la responsabilité des Etats, voir CRAWFORD, James, OLLESON, Simon. The Continuing Debate on a UN Convention on State Responsibility. In: International and Comparative Law Quarterly, 54 
Or, il est clair que ces arguments, favorables à l'adoption de l'approche non-conventionnelle de la codification par la CDI, ne sauraient s'appliquer avec la même force et les mêmes effets à l'ensemble des textes composant la catégorie des instruments non-contraignants. L'impact de ces arguments serait évident et presque escompté par rapport aux instruments dont le but essentiel est d'élucider les aspects techniques de fonctionnement du droit international (conclusions sur la Fragmentation du droit international), de compléter et intégrer une discipline conventionnelle de codification déjà existante (guide de la Pratique des réserves aux traités), d'en éclairer l'interprétation ou les aspects problématiques de mise en œuvre (conclusions sur les Accords et pratiques ultérieurs dans l'interprétation des traités), ou de fournir des instruments destinés à faciliter la tâche des opérateurs juridiques (conclusions sur la Détermination du droit international contumier). Comme on l'a déjà remarqué, ces textes sont sensés servir des finalités immédiates d'ordres pratique, technique ou pédagogique, par rapport auxquelles l'adoption $\mathrm{du}$ format contraignant ne saurait pas, en soi, apporter grand-chose. Au contraire, l'instrument non-conventionnel et les exigences de rapidité et de flexibilité qui y sont associés sembleraient aptes à garantir le bon succès de l'œuvre de codification portant sur les sujets " techniques» susmentionnés ${ }^{41}$.

Plus délicate paraît par contre la tâche d'évaluer le bien fondé des arguments favorables au format non conventionnel par rapport à la typologie des projets d'articles de la Commission ayant une vocation plus strictement normative, c'està-dire sensés codifier les règles coutumières substantielles applicables dans un certain domaine du droit international. C'est en effet par rapport à cette typologie de règles que les exigences de certitude juridique, qui sembleraient assurées par la transposition dans un texte contraignant, se présentent d'autant plus fortes et impérieuses. Face à ces exigences, on pourrait se demander si la tendance de la CDI consistant à généraliser le recours au format non contraignant est susceptible de conduire à long terme au succès du processus de codification ou, au contraire, déterminer son échec.

Il est intéressant à cet égard de revenir sur la réponse, élaborée initialement en référence au projet d'articles sur la responsabilité internationale de l'Etat ${ }^{42}$, qui consiste à laisser ouverte la question du format final - conventionnel ou non d'un projet d'articles donné, mais qui vise en même temps à évaluer l'impact que ce texte est à même de produire sur la pratique internationale, en prenant tout

(2005), pp. 959-972.

41 Voir à ce sujet les remarques de PELLET. Conclusions... cit., p. 337.

42 Voir les résolutions de l'AG 59/35 du 2 décembre 2004, 62/61 du 6 décembre 2007, 65/19 du 6 décembre 2010,68/104 du 16 décembre 2013. Une telle réponse commence par contre à être " exportée " aussi par rapport à d'autre textes de la CDI, comme celui sur la responsabilité des Organisations internationales (voir la résolution de l'AG 69/126 du 10 décembre 2014) ou celui concernant la prévention des dommages transfrontières résultant d'activités dangereuses (voir la résolution de l'AG 68/114 du 16 décembre 2013). 
particulièrement comme terme de référence son utilisation dans la jurisprudence internationale. Il est bien connu que cette approche s'est traduite, dans le cas du projet sur la responsabilité internationale de l'Etat, par la rédaction d'une compilation présentée par le Secrétaire général des Nations Unies en 2007, et depuis actualisée en 2010 et 2013, faisant état d'une ample application judiciaire des articles de la $\mathrm{CDI}^{43}$. Ce résultat a permis d'étayer l'argument selon lequel les projets d'articles de la CDI seraient à même d'exercer une influence importante dans la pratique des relations internationales nonobstant leur statut d'actes non contraignants, et démontrerait finalement le succès de l'approche à la codification menée par l'entremise d'instruments non conventionnels ${ }^{44}$.

On pourrait observer que la tentative de consolider l'impact d'un projet d'articles de la CDI au travers de la " mise à l'épreuve de la pratique », tout en étant intéressant et significatif, se présente toutefois entaché par certains limites structurelles importantes. En référence au cas du texte sur la responsabilité internationale de l'Etat, la plus remarquable de ces limites est révélée par le fait que la compilation de la pratique judiciaire témoigne d'une utilisation fort inégale des articles du projet, ce qui semble conditionner la possibilité de tirer des conclusions généralisées quant au statut et à l'autorité de l'instrument dans son ensemble ${ }^{45}$. A ce sujet, l'on pourrait prendre comme indice révélateur l'utilisation très circonstanciée des articles de la CDI sur la responsabilité étatique qui a été faite dans la jurisprudence récente de la Cour internationale de Justice ${ }^{46}$. On peut à cet égard relever que certaines des dispositions contenues dans la première partie du projet d'articles, concernant les principes généraux en matière d'attribution du comportement à l'Etat ${ }^{47}$, de la responsabilité à raison du fait d'un autre

43 Voir Compilation de décisions de juridictions internationales et d'autres organes internationaux. Rapport du Secrétaire général, UN doc. A/62/62 du $1^{\text {er }}$ février 2007, et Addendum 1 du 17 avril 2007 ; UN doc. A/65/76 du 30 avril 2010 ; UN doc. A/68/72 du 30 avril 2013. L'ensemble de ces documents fait état de 212 cas dans lesquels les articles de la CDI ont été cités par des organes judiciaires ou parajudiciaires internationaux.

44 Voir par exemple BOYLE, Alan, CHINKIN, Christine. The Making of International Law. Oxford: Oxford University Press, 2007, pp. 183-185; VILLALPANDO. Codification Light... cit., pp. 144-145.

45 Pour un exposé plus détaillé de cet argument, on se permet de renvoyer à ARCARI, Maurizio. Le juge et la codification du droit de la responsabilité. Quelques remarques concernant l'application judiciaire des articles de la CDI sur la responsabilité de l'Etat pour fait internationalement illicite. In : BOSCHIERO, Nerina, SCOVAZZI, Tullio, PITEA, Cesare, RAGNI, Chiara (eds.). International Courts and the Development of International Law. Essays in Honour of Tullio Treves. The Hague: T.M.C. Asser Press/Springer, 2013, pp. 19-30.

46 Sur la question en général, voir VILLALPANDO, Santiago. Le codificateur et le juge face à la responsabilité internationale de l'Etat : interaction entre la CDI et la CIJ dans la détermination des règles secondaires. In : Annuaire Français de Droit International, 55 (2009), p. 39 s.

47 Voir par exemple, Affaire relative à l'application de la Convention pour la prévention et la répression du crime de génocide (Bosnie-Herzégovine c. Serbie-et-Monténégro), arrêt du 26 février 2007. In : CIJ Recueil, 2007, respectivement p. 202, par. 385, et p. 207, par. 398, où les règles énoncées aux articles 4 et 8 du projet sur la responsabilité en matière d'attribution 
$E^{2} t^{48}$, ou de circonstances excluant l'illicéité ${ }^{49}$, ont été expressément citées par la Cour comme reflétant le droit coutumier existant. Par ailleurs, dans d'autres occasions, concernant l'application des principes en matière de conséquences de violations graves d'obligations internationales d'importance fondamentale ${ }^{50} \mathrm{ou}$ d'invocation de la responsabilité internationale ${ }^{51}$, la Cour, tout en évoquant dans son raisonnement la logique des solutions retenues par la CDI, s'est abstenue de renvoyer de manière explicite aux dispositions pertinentes du projet sur la responsabilité de l'Etat. Il n'est pas surprenant pas que cette attitude prudente de la Cour se soit manifestée par rapport aux dispositions du projet sur la responsabilité qui ont été l'objet d'un accueil réservé par les Etats et dont la correspondance au droit coutumier positif se présente toujours comme une question ouverte.

Il semble en somme que, tout en étant importantes et révélatrices, les réactions provenant d'un public d'utilisateurs privilégiés des articles de la CDI,

à l'Etat du comportement de ses organes et du comportement de personnes privées agissant sous la direction ou le contrôle de l'Etat ont été qualifiées par la Cour comme relevant du droit international coutumier.

48 Voir ibidem, p. 217, par. 420, où la règle énoncée à l'art. 16 du projet sur la responsabilité, concernant l'aide ou l'assistance dans la commission du fait internationalement illicite, a été qualifiée par la Cour comme exprimant une règle coutumière.

49 Voir Affaire relative au projet Gabčíkovo-Nagymaros (Hongrie/Slovaquie), arrêt du 25 septembre 1997. In : CIJ Recueil, 1997, p. 40, par. 52, où les conditions pour l'invocation de la nécessité prévues par l'art. 33 (actuellement art. 25) du projet sur la responsabilité ont été qualifiées par la Cour comme reflétant le droit international coutumier.

50 Voir Conséquences juridiques de l'édification d'un mur dans le territoire palestinien occupé, avis consultatif du 9 juillet 2004. In : CIJ Recueil, 2004, p. 200, par. 159, où la Cour, après avoir relevé la nature et l'importance des obligations internationales violées, a affirmé l'engagement de tous les Etats de ne pas reconnaître la situation illicite découlant de la construction du mur et de ne pas prêter assistance à Israël dans le maintien de la situation créée par cette construction : à l'occasion, la Cour a donc paru faire application des conséquences particulières découlant de la violation d'une obligation posée par des normes impératives du droit international, telles qu'énoncées à l'art. 41 du projet de la CDI, sans faire référence directe à cette disposition. Plus récemment, dans l'affaire des Immunités juridictionnelles de l'Etat (Allemagne c. Italie; Grèce intervenant), arrêt du 3 février 2012. In : CIJ Recueil, 2012, p. 140, par. 93, la Cour a affirmé que le fait de reconnaitre l'immunité d'un Etat étranger conformément au droit international coutumier ne revient pas à juger licite une situation créée par la violation d'une règle de jus cogens, ni à prêter aide ou assistance au maintien de cette situation, et ne saurait donc contrevenir " au principe énoncé par l'art. 41 des articles de la Commission du droit international sur la responsabilité de l'Etat ". Tout en évoquant expressément la disposition, la Cour s'est toutefois abstenue de se prononcer sur la conformité au droit international coutumier du principe y énoncé.

51 Voir Questions concernant l'obligation de poursuivre ou d'extrader (Belgique/Sénégal), arrêt du 20 juillet 2012. In : CIJ Recueil, 2012, pp. 449-450, par. 68-69, où la Cour a déclaré la nature d'obligations erga omnes partes des dispositions de la Convention de 1980 contre la torture, et en a tiré comme conséquence que " tout Etat partie à la convention contre la torture peut invoquer la responsabilité d'un autre Etat partie dans le but de faire constater le manquement allégué de celui-ci à des obligations erga omnes partes ". Tout en évoquant la logique de l'art. 48, par. 1, du projet sur la responsabilité des Etats, concernant la problématique de l'invocation de la responsabilité par un Etat autre qu'un Etat lésé, la Cour s'est néanmoins abstenue de se référer expressément à cette disposition. 
tels que les juges internationaux, sauraient difficilement pallier l'autorité des maîtres ultimes du processus de codification, qui sont les Etats. En effet, c'est justement pour sortir des incertitudes de la pratique (et de l'opinio iuris) que l'œuvre de codification est entamée, et un moment décisif dans ce passage est représenté par l'expression de la volonté des Etats de se lier à une règlementation conventionnelle spécifique.

A cet égard, il ne semble pas superflu d'évoquer la distinction, très floue mais pourtant essentielle, entre codification et développement progressif du droit international, qui demeure au cœur de chaque entreprise de codification. S'il est vrai que les aspects de la codification et du développement progressif se trouvent souvent entremêlés dans les projets de la CDI, il est aussi vrai que le différent dosage entre les deux volets pourrait avoir un impact sur le choix du format approprié pour l'instrument de codification. Il faut ne pas oublier que, aux termes du Statut de la CDI, le format conventionnel était expressément prévu pour le traitement des sujets " non encore réglés par le droit international ou relativement auxquels le droit n'est pas encore suffisamment développé dans la pratique des Etats " ${ }^{52}$, tandis que les issues " non-contraignantes » étaient plutôt envisagées pour la systématisation des règles du droit international relevant des domaines où une pratique étatique et des précédents nombreux existaient déjà (c'est-à-dire, la codification au sens strict $)^{53}$. Si cette distinction technique a été largement dépassée dans la pratique de la CDI, il reste qu'un nombre considérable de commentateurs tombe d'accord sur la conclusion de principe selon laquelle le développement progressif du droit international devrait se faire en premier lieu par l'entremise d'instruments conventionnels ${ }^{54}$, car seulement ces derniers seraient à même de conférer aux textes élaborés par la CDI l'autorité de la règle de droit ${ }^{55}$.

Pour revenir à notre point, l'on pourrait dire que si l'exercice de la CDI consiste à interpréter, éclairer ou consolider les aspects techniques d'un certaine domaine du droit international, la plus grande souplesse de formes sera admissible et le format non conventionnel sera susceptible de servir les finalités en jeu ; si, au contraire, la détermination du contenu et de la portée de règles nouvelles ou en voie de formation du droit international est en cause, la confirmation de la valeur normative des solutions proposées par la Commission dans un instrument

52 Voir l'art. 15 du Statut de la CDI, ainsi que les articles 16 et 17, dédiés à la procédure que la CDI devait suivre dans le cas de renvoi par l'AG de propositions concernant le développement progressif du droit international.

53 Voir les alternatives mentionnées par l'art. 23 du Statut de la CDI, cit. supra, note 13, qui est compris dans la section du Statut dédiée à la "Codification du droit international ".

54 Voir MURPHY, Sean D. Codification, Progressive Development, or Scholarly Analysis? The Art of Packaging the ILC's Work Product. In: RAGAZZI, Maurizio (ed.). The Responsibility of International Organizations. Essays in Memory of Ian Brownlie. Leiden/Boston: Brill Publisher, 2013, p. 30: "Progressive development could only be done by means of a convention ".

55 Cf. LUSA BORDIN. Reflections..., cit., p. 560. 
contraignant, soutenu et accepté par l'expression de la volonté des Etats de s'engager, se révélera probablement incontournable. Dans cette perspective, il paraît difficile d'admettre que le format non-conventionnel puisse répondre aux exigences de certitude juridique, qui caractérisent le traité, et que celui-ci se trouve complètement et définitivement supplanté en tant qu'instrument de codification (au sens large) du droit international.

\section{LA «CODIFICATION MOLLE » DU DROIT INTERNATIONAL ET LE ROLE DE LA CDI DANS CE DOMAINE}

En écrivant en 1957 un article sur la vocation de la CDI, Julius Stone manifestait ses perplexités sur le processus de codification du droit international et plaidait pour la transformation de la CDI en un centre de recherche en droit international, chargé d'étudier les problèmes basiques de la matière et d'élaborer un programme ample et flexible visant à la meilleure compréhension $\mathrm{du}$ fonctionnement de l'ordre juridique international ${ }^{56}$. Comme noté par un commentateur avisé des travaux de la CDI, la position à la fois sceptique et visionnaire de Julius Stone était probablement influencée par la circonstance que, dans la période historique dans lequel l'éminent auteur écrivait - celle de la « guerre froide » de fin années 50 -, aucun des projets à l'attention de la Commission n'avait pas encore été transformé en convention ${ }^{57}$. Néanmoins, il est intéressant de constater que dans les actes d'un colloque des Nations Unies sur le développement progressif et la codification du droit international parus plus de 40 ans plus tard, la proposition selon laquelle « la Commission devrait contribuer à orienter le processus d'édification du droit international » figure à la première place dans la liste d'idées et suggestions visant à renforcer le rôle de la $\mathrm{CDI}^{58}$. A côté de cette première proposition générale, qui évoque l'idée d'un observatoire mondial du droit international déjà avancée par Julius Stone, dans la suite de la liste des suggestions du Colloque se trouvent aussi détaillées les

56 Voir STONE, Julius. On the Vocation of the International Law Commission. In: Columbia Law Review, 18 (1957), p. 16 s, spéc. pp. 49-51: "Its [ILC’s] broad new mandate would be to plan, to supervise, and, in part, to carry out a wide and flexible program aimed at extending our knowledge and understanding of the operation of international law as a means of social control».

57 SINCLAIR. The International Law Commission..., cit., p. 138.

58 Voir Pour un meilleur droit international: la Commission du droit international à 50 ans. Actes $d u$ Colloque des Nations Unies sur le développement progressif et la codification $d u$ droit international. New York : Nations Unies, 1998, pp. 48-65, sous la rubrique « Idées et suggestions visant à renforcer la Commission du droit international et à accroître sa capacité de développement progressif et de codification du droit international ", spéc. à p. 48 : " La Commission devrait donner son avis et formuler des recommandations sur les initiatives qui s'inscrivent dans le processus d'édification du droit international, en étudiant le droit international et les relations internationales en vue de cerner les domaines pour lesquels il serait opportun que la Commission elle-même ou un autre organe normatif international élabore une règlementation ". 
différentes formes que pourraient prendre les travaux de la CDI et, de manière significative, un bon nombre d'instruments et de formats non-conventionnels sont mentionnés ${ }^{59}$.

Or si, comme on l'a très justement noté, la suggestion concernant le rôle de la CDI comme édificateur du droit international relève probablement d'une " ambition hors portée ${ }^{60}$, il est indiscutable - et les instruments non conventionnels répertoriés au cours de la présente analyse sont là pour le confirmer - que la Commission a été parfaitement à la hauteur des auspices du colloque des Nations Unies pour ce qui est de la mise à jour de ses instruments de travail. Par ailleurs, on ne saurait exclure qu'une liaison existe entre les deux aspects susmentionnés et qu'elle soit tout à fait pertinente à nos fins actuelles.

On pourrait en effet observer que, sur un plan général, les instruments nonconventionnels ont représenté les moyens à disposition de la CDI pour canaliser dans le domaine de la codification certaines des tendances clés qui caractérisent le droit international moderne. Il en va ainsi, tout d'abord, pour la diffusion d'une approche souple et flexible de la production normative, qui se réalise dans certains domaines du droit international à travers les instruments typiques de la "soft law », tels que les lignes directrices, les directives non contraignants ou les déclarations de principe ${ }^{61}$. De ce point de vue, le fait que la Commission, dès le début de l'étude sur le sujet de la Protection de l'atmosphère, ait envisagé pour l'issue de ses travaux la forme du projet de directives ${ }^{62}$, et la circonstance qu'au moins cette orientation n'ait pas été l'objet d'objections substantielles de la part des Etats réunis à la Sixième Commission de l'AG ${ }^{63}$, ne fait que confirmer l'emprise que les instruments de la « soft law » sont appelés à déployer dans des domaines spécifiques du droit international, tels que celui de la protection de l'environnement.

D'autre part, on peut aussi ajouter que l'emploi des instruments nonconventionnels a permis à la Commission d'adresser ses études à un public d'utilisateurs plus vaste par rapport à celui constitué par les traditionnels

59 Ibidem, p. 58, où sous la rubrique «Les travaux de la Commission et la mise en forme du droit international » les instruments suivants sont mentionnés : "Des projets de convention-cadre ; des traités types; des règles types ; des lois types devant être adoptées à l'échelon international ; des déclarations de principe; de nouvelles formulations (restatements) des règles de droit ; des codes de conduite ; des directives ; des guides d'application à l'intention des Etats ».

60 Cf. PELLET. La Commission du droit international... cit., p. 609.

61 Sur l'importance de la "soft law » comme moyen de production normative dans le droit international contemporain, voir notamment BOYLE, CHINKIN. The Making of International Law... cit., pp. 211-229.

62 Voir Rapport de la Commission du droit international, Soixante-cinquième session 6 mai-7 juin et 8 juillet-9 août 2013, UN doc. A/68/10 (2013), par. 168, lettre d).

63 Voir Résumé thématique... cit., par. 27-33. Ce résumé des débats à la Sixième Commission, tout en faisant état des difficultés qui accompagnent le sujet de la protection de l'atmosphère, néanmoins ne révèle pas des critiques importantes à l'approche «molle » choisie par la CDI pour la forme finale de ses travaux. 
destinataires du processus de codification, c'est-à-dire les Etats. A cet égard, l'on ne saurait qu'avaliser le constat formulé par un observateur des travaux de la Commission, selon lequel « the ILC is envisioning for itself a wider audience, one made up not just for States, but also of attorneys, judges, policymakers, and academics ${ }^{64}$. De ce point de vue, l'attention réservée à la réception des textes élaborés par la CDI dans la pratique judiciaire (dont on a auparavant mentionné un exemple au sujet de la responsabilité internationale de l'Etat), ainsi que la volonté d'élaborer des instruments opérationnels à l'intention des opérateurs du droit international (qui a été avalisée dans les contexte des conclusions sur la fragmentation du droit international ou du projet de conclusions sur la détermination du droit coutumier), ne sont que quelques signaux confirmant la sensibilisation croissante de la Commission à l'égard du rôle des acteurs nonétatiques dans le processus de la production normative internationale ${ }^{65}$. Les instruments non-conventionnels proposés par la CDI semblent donc confirmer et relancer les considérations formulées par Yves Daudet, lequel, dans la conclusion de son cours à l'Académie de La Haye sur la codification du droit international, notait que « [l]a Commission, dans ces toutes dernières années, donne ainsi quelques signes d'adaptation aux changements et de capacité à nourrir la souplesse et la plasticité du droit international ${ }^{66}$.

Tout cela étant dit quant aux potentialités des instruments nonconventionnels de codification, il reste néanmoins à établir quelle serait leur contribution à la véritable finalité de l'œuvre de codification, qui demeure en dernière analyse de consolider et augmenter la certitude de la règle coutumière. A ce sujet, on ne peut que reprendre les considérations déjà esquissées concernant les dangers d'une «perte d'autorité » des résultats du processus de codification, qui serait la conséquence du statut d'actes non-contraignants des instruments non-conventionnels et de la circonstance qu'ils amènent fatalement à contourner l'expression de la volonté des Etats de se lier à une certaine proposition normative ${ }^{67}$. A ce stade, on pourrait aussi mentionner en passant un autre effet

64 Cf. KATZ COGAN. The Changing Form... cit., pp. 284.

65 Sur les termes généraux de la problématique voir ARCARI, Maurizio. Remarques sur les dynamiques entre sujets et normativité en droit international. In : ARCARI, Maurizio, BALMOND, Louis (dir.). Diversification des acteurs et dynamiques normatives en droit international. Napoli : Editoriale Scientifica, 2013, pp. 9-30, ainsi que les rapports réunis dans l'ouvrage collectif mentionné.

66 Cf. DAUDET Yves. Actualités de la codification du droit international. In : Recueil des Cours de l'Académie de Droit International, 303 (2003), p. 116.

67 Voir supra, section 3, et aussi les considérations de VILLALPANDO. Codification Light... cit., p. 151, lequel souligne que " this process may take place outside the ultimate control of Governments and may lead to the crystallization of opinion iuris simply by the mutual reaffirmation of the existence of a norm by codifiers, judges and academics. In other words, the role played by States, both through international negotiations in treaty making and through their practice in customary international law, is transformed-and may even be circumventedby a 'codification light' ". 
négatif que les instruments non-conventionnels de codification risqueraient de se voir attribuer par les Etats eux-mêmes : ceux-ci pourraient en effet être tentés d'associer l'emploi de ces instruments à des projets de codification considérés insatisfaisants en raison de leur prétendue non-conformité au droit coutumier, en de reléguer de cette manière les textes proposés par la CDI dans les limbes des actes dépourvus d'effets juridiques ${ }^{68}$. Dans cette perspective, les instruments non conventionnels seraient susceptibles de convoiter dans notre domaine le même effet de "décodification » du droit international qu'ils étaient censés pallier ${ }^{69}$. De ce fait, l'effort de codification mené par la CDI et sa contribution à l'édification du droit international risqueraient d'être reléguées à l'arrière-plan, avec toutes les conséquences négatives qui en découleraient sur le plan de la certitude juridique.

En conclusion, on ne saurait certainement pas nier les vertus des instruments non-conventionnels, lesquels à la fois confirment la pluralité de possibilités disponibles à la CDI pour achever sa tâche de codification et développement progressif du droit international et permettent d'actualiser et dynamiser le rôle de la Commission dans ce domaine. Dans le même temps, on ne saurait pas non plus souscrire à la suggestion mentionnée au début de cette contribution, selon laquelle les instruments non-conventionnels auraient définitivement supplanté les traités dans la codification du droit international. Etant donnée la complexité des exigences qui sont à la base de l'œuvre de codification, prétendre faire confiance de manière exclusive sur un seul format pour déterminer son issue semblerait

68 On peut trouver des exemples de cette tendance dans les remarques formulées par les Etats à la Sixième Commission de l'AG en 2014, au sujet du format de certains projets d'articles à l'étude de la CDI. De l'avis de la délégation de la Fédération de Russie, « en ce qui concerne le sujet 'Protection des personnes en cas de catastrophes' (...) les résultats finals devraient être des directives et non un projet d'articles. La CDI élabore des projets d'articles sur des sujets qui, étant donné l'existence d'une pratique des Etats bien développée, se prêtent à la formulation d'un instrument juridiquement contraignant. La délégation russe ne considère pas que le sujet à l'examen s'y prête. (...) Les règles formulées dans le projet d'articles ne doivent pas être considérées comme des normes du droit international coutumier, mais comme un exemple de développement progressif du droit " (voir UN doc. A/C.6/SR.19, par. 96-97 - les italiques sont ajoutées). Dans le même sens, intervenant sur le projet de la CDI sur l'expulsion des étrangers, le délégué du Royaume-Uni a observé que «La position du Royaume-Uni a toujours été que la CDI devrait mettre terme à ses travaux sur le sujet et ne pas aller plus loin. Il continue de penser que l'expulsion des étrangers n'est pas un sujet qui se prête à l'élaboration d'une convention : il ne considère pas que le projet d'articles reflète le droit international coutumier, et il n'est pas d'accord avec les articles qui prétendent représenter un développement progressif du droit international. (...) Quant à la forme du résultat éventuel des travaux de la CDI, des directives susceptibles de promouvoir de bonnes pratiques seraient plus utiles aux Etats qu'un instrument juridiquement contraignant » (ibidem, par. 165-166 - italiques ajoutées). Il est juste la peine de noter que ces arguments se basent apparemment sur le renversement de la logique qui était propre au Statut de la CDI, selon laquelle le format conventionnel serait tout spécialement demandé pour les projets d'articles relevant davantage du développement progressif du droit international (cf. supra, le texte relatif aux notes 51-52). Sur les risques de la "dilution normative " découlant de la classification des textes de la CDI comme instruments de pur «droit mou » voir les remarques de PRONTO. Some Thoughts... cit., pp. 613-615.

69 Voir supra, le texte relatif à la note 36. 
illusoire, aussi bien que dangereux. C'est plutôt dans l'utilisation sage et dans le dosage savant entre instruments conventionnels et non-conventionnels que paraissent résider les chances du succès de l'œuvre de codification menée par la CDI, ainsi que du prestige et de l'autorité de cette vénérable institution. 


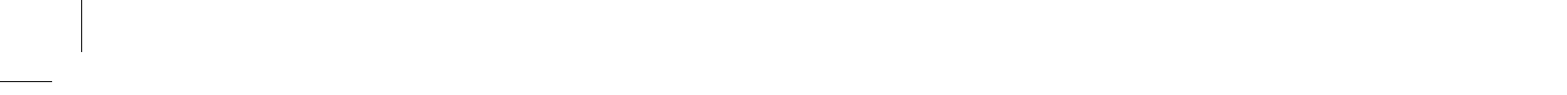

